\title{
Transplante de córnea em um hospital de referência no norte do Brasil
}

\author{
Corneal transplant at a reference hospital in Northern Brazil \\ Trasplante de córnea en un hospital de referencia en el norte de Brasil
}

Pablo de Melo Maranhão Pereira ${ }^{1 *}$, José Jesu Sisnando D’Arauj Filho1, Raissa Tereza Casseb Oliveira $^{1}$, Olga Tem Caten Pies Lameira ${ }^{1}$, Fernanda Braga Cordeiro Franco Rodrigues ${ }^{1}$, Maria Elisa Monteiro de Freitas ${ }^{1}$, Leonardo Cruz Xavier ${ }^{1}$.

\section{RESUMO}

Objetivo: Este estudo avaliou as principais causas de indicação do transplante de córnea no Hospital Universitário Bettina Ferro de Souza, do período de outubro de 2011 a dezembro de 2017. Métodos: Realizou-se coleta de dados clínico-epidemiológicos como: gênero, faixa etária e procedência (capital ou interior), além de dados oftalmológicos como: etiologia da indicação, tempo de espera para o procedimento cirúrgico, tipo de procedimento (se eletivo ou urgente) de todos os pacientes submetidos ao transplante de córnea realizados no Hospital Universitário Bettina Ferro de Souza (HUBFS). Resultados: A maioria dos pacientes é do sexo masculino, com faixa etária maior que 60 anos. Houve um pequeno predomínio de indivíduos do interior do Estado e outras cidades do norte do Brasil, sendo a maior parte dos procedimentos realizados em caráter de urgência. A úlcera de córnea foi a principal indicação para o transplante de córnea, seguida por leucoma e ceratopatia bolhosa. Quanto ao tempo de espera para o procedimento de critério urgente, observou-se que mais de três quartos dos pacientes foram submetidos ao transplante num período de até 14 dias e nas indicações eletivas houve uma espera média de dois anos e dois meses. Conclusão: Conclui-se então que as principais causas de indicação do transplante de córnea no Hospital Universitário Bettina Ferro de Souza, do período de outubro de 2011 a dezembro de 2017, foram úlcera de córnea, seguida por leucoma e ceratopatia bolhosa.

Palavras-chave: Transplante de córnea, Transplante de urgência, Úlcera de córnea.

\begin{abstract}
Objective: This study evaluated the main causes of corneal transplantation in Bettina Ferro de Souza University Hospital, from October 2011 to December 2017. Methods: Clinical and epidemiological data were collected, such as gender, age group and origin (capital or countryside), in addition to ophthalmologic data such as: etiology of the indication, waiting time for the surgical procedure, type of procedure (if elective or urgent) of all patients submitted to corneal transplantation performed at Bettina Ferro de Souza University Hospital (HUBFS). Results: The majority of patients are male, with an age group of over 60 years. There was a small predominance of individuals from the countryside of the State and other cities in the North of Brazil, most of the procedures being carried out as a matter of urgency. Corneal ulcer was the main indication for corneal transplantation, followed by leukoma and bullous keratopathy. Regarding the waiting time for the urgent procedure, it was observed that more than three-quarters of the patients underwent transplantation within a period of up to 14 days and in the elective indications there was an average wait of two years and two months. Conclusion: It was concluded that the main causes of cornea transplantation in Bettina Ferro de Souza University Hospital, from October 2011 to December 2017, were corneal ulcer, followed by leukoma and bullous keratopathy.
\end{abstract}

Keywords: Corneal transplantation, Emergency transplantation, Corneal ulcer.

${ }^{1}$ Hospital Universitário Bettina Ferro de Souza. Belém-Pará. *E-mail: pablomaranhao3@hotmail.com 


\begin{abstract}
RESUMEN
Objetivo: Este estudio evaluó las principales causas de indicación del trasplante de córnea en el Hospital Universitario Bettina Ferro de Souza, del período de octubre de 2011 a diciembre de 2017. Métodos: Se realizó la recolección de datos clínico-epidemiológicos como: género, grupo de edad y procedencia (capital o interior), además de datos oftalmológicos como: etiología de la indicación, tiempo de espera para el procedimiento quirúrgico, tipo de procedimiento (si electivo o urgente) de todos los pacientes sometidos al trasplante de córnea realizados en el Hospital Universitario Bettina Ferro de Souza (HUBFS). Resultados: La mayoría de los pacientes es del sexo masculino, con un grupo de edad mayor a 60 años. Se observó un pequeño predominio de individuos del interior del Estado y otras ciudades del norte de Brasil, siendo la mayor parte de los procedimientos realizados en carácter de urgencia. La úlcera de córnea fue la principal indicación para el trasplante de córnea, seguida por leucoma y queratopatía bullosa. En cuanto al tiempo de espera para el procedimiento de criterio urgente, se observó que más de tres cuartos de los pacientes fueron sometidos al trasplante en un período de hasta 14 días y en las indicaciones electivas hubo una espera promedio de dos años y dos meses. Conclusión: Se concluye entonces que las principales causas de indicación del trasplante de córnea en el Hospital Universitario Bettina Ferro de Souza, del período de octubre de 2011 a diciembre de 2017, fueron úlcera de córnea, seguida por leucoma y ceratopatía bullosa.
\end{abstract}

Palabras clave: Trasplante de córnea, Trasplante de urgencia, Úlcera de córnea.

\title{
INTRODUÇÃO
}

Afecções graves corneanas estão entre as maiores causas de cegueira do mundo, atrás apenas de catarata, glaucoma e degeneração macular relacionada à idade (SMINKEY L, 2018). Cerca de 5 milhões de pessoas apresentam cegueira unilateral de causa corneana por ano, sendo que as principais causas são cicatrizes de tracoma, trauma ocular, ceratites e, ainda, ceratopatia bolhosa pós-cirurgia de catarata, ceratocone e condições hereditárias (distrofias) (SMINKEY L, 2018; BENTLEY TS, et al., 2017).

Dentre as opções de tratamento comum a tais condições, há o transplante de córnea nas suas mais variadas técnicas, seja esse penetrante ou lamelar. Devido à melhoria nas técnicas operatórias e de coleta/preservação do órgão, associada à característica avascular do tecido e, por conseguinte, maior conhecimento e condução de imunodepressores e corticoterapias evitando rejeição ou mesmo falência, o transplante de córnea tornou-se o procedimento mais realizado no mundo entre todas as modalidades de transplante (BENTLEY TS, et al., 2017; EYE BANKING STATISTICAL, 2016).

No Brasil, a realidade mundial se espelha nisso, apresentando nas últimas décadas um aumento do número de transplantes por ano, o que é justificado pela abertura de mais bancos de olhos e de serviços que façam tal procedimento, seguindo, inclusive, as estatísticas americanas, nas quais se observou aumento do número de procedimentos em $5 \%$, de 2005 a 2014 (BENTLEY TS, et al., 2017; EYE BANKING STATISTICAL, 2016; CRUZ GK, et al., 2016).

O transplante de córnea penetrante ainda é a técnica mais realizada em todo o mundo, inclusive, no Brasil, onde a Região Sudeste se apresenta com o maior número de transplantes do país (CRUZ GK, et al., 2017; NEVES RC, et al., 2010; Chen MC, et al., 2017).

No entanto, o Estado do Pará figura entre as primeiras dez unidades federativas em quantidade de realização do procedimento, já tendo realizado 13 cirurgias somente no mês de março de 2018, segundo dados do Datasus (MINISTÉRIO DA SAÚDE, 2018).

Todavia, poucas são as publicações na literatura sobre tal tema em relação à Região Amazônica, tendo o último registro sido feito há mais de sete anos. ${ }^{(11)}$ Some-se a isso que o Hospital Universitário Bettina Ferro de Souza é o centro de referência regional para afecções oftalmológicas, incluindo o transplante de córnea, tendo realizado mais de cem transplantes no último ano (DEKARIS L, 2013). 
Dessa forma, torna-se necessário traçar o perfil clínico-epidemiológico e oftalmológico dos pacientes submetidos ao transplante de córnea no hospital de referência do Estado e da Região, e as principais indicações desse procedimento.

\section{MÉTODOS}

Trata-se de um estudo retrospectivo e quantitativo, no qual se realizou coleta de dados de todos os pacientes submetidos ao transplante de córnea realizados no Hospital Universitário Bettina Ferro de Souza (HUBFS). A coleta de dados ocorreu após aprovação do projeto de pesquisa no Comitê de Ética em Pesquisa (número do protocolo 1234680) e assinatura do Termo de Consentimento Livre e Esclarecido (TCLE) pelos participantes do estudo, com data de novembro de 2018. Ainda para realização da pesquisa, solicitou-se autorização à direção clínica do HUBFS e aceite do orientador do estudo. Todos os pacientes submetidos ao transplante no período estipulado foram contatados via telefone adquirido em suas fichas de inscrição na fila de transplante e/ou na ficha-cadastro de registro do hospital, para a solicitação da presença de seus dados clínicos no projeto de pesquisa.

Foram incluídos todos os pacientes submetidos ao procedimento cirúrgico no HUBFS do período de outubro de 2011 a dezembro de 2017, sem restrição ao gênero, faixa etária, tipo de indicação, comorbidade sistêmica ou ocular e que aceitaram participar do estudo com prévia assinatura do Termo de Consentimento Livre e Esclarecido. Em contrapartida, foram excluídos aqueles que não quiseram assinar o TCLE ou que não foram submetidos ao procedimento, mesmo estando ainda cadastrados na fila de transplante do Estado local.

Coletaram-se dados clínico-epidemiológicos, como: gênero, faixa etária e procedência (capital ou interior). Além de dados oftalmológicos como: etiologia da indicação, tempo de espera para o procedimento cirúrgico, tipo de procedimento (se eletivo ou urgente). Tais dados foram agrupados em protocolo de pesquisa e coletados no mês de novembro de 2018, para, então, serem submetidos à análise estatística com o programa Biostat, utilizando o método T-student, considerando nível de significância com $p<0,05$.

\section{RESULTADOS}

Foi observada uma maior população de pessoas do sexo masculino dentre toda a séria histórica dos transplantes de córnea no HUBFS comportando-se uma porcentagem de 57,1 do total. Em relação à faixa etária, a maioria absoluta foi de pacientes com idade acima de sessenta anos (32,3\%), apresentando também um predomínio em paciente das faixas de 31 a 40 e 41 a 50, obtendo-se uma idade média de 46 anos. Quanto à procedência, percebeu-se um pequeno predomínio $(52,1 \%)$ de indivíduos do interior do Estado do Pará e outras cidades do Norte do Brasil (Tabela 1).

Tabela 1 - Caracterização dos pacientes submetidos ao transplante de córnea no Hospital Universitário Bettina Ferro de Souza no período de outubro de 2011 a dezembro de 2017.

\begin{tabular}{|c|c|c|}
\hline Variável & $\mathbf{N}$ & $\%$ \\
\hline \multicolumn{3}{|l|}{ Sexo } \\
\hline Masculino & 108 & 57,1 \\
\hline Feminino & 81 & 42,9 \\
\hline \multicolumn{3}{|l|}{ Idade } \\
\hline $0-4$ & 1 & $0,5 \%$ \\
\hline $5-10$ & 8 & $4,2 \%$ \\
\hline $11-20$ & 13 & $6,9 \%$ \\
\hline $21-30$ & 23 & $12,2 \%$ \\
\hline $31-40$ & 32 & $16,9 \%$ \\
\hline $41-50$ & 33 & $17,5 \%$ \\
\hline $51-60$ & 18 & $9,5 \%$ \\
\hline$>60$ & 61 & $32,3 \%$ \\
\hline \multicolumn{3}{|l|}{ Procedência } \\
\hline Capital & 83 & $43,9 \%$ \\
\hline Interior & 106 & $52,1 \%$ \\
\hline Total & 189 & - \\
\hline
\end{tabular}


A úlcera corneana foi a principal indicação para o transplante de córnea na amostra, seguida por leucoma e ceratopatia bolhosa (Gráfico 1).

Gráfico 1 - Indicação dos transplantes de córnea realizados no Hospital Universitário Bettina Ferro de Souza no período de outubro de 2011 a dezembro de 2017.

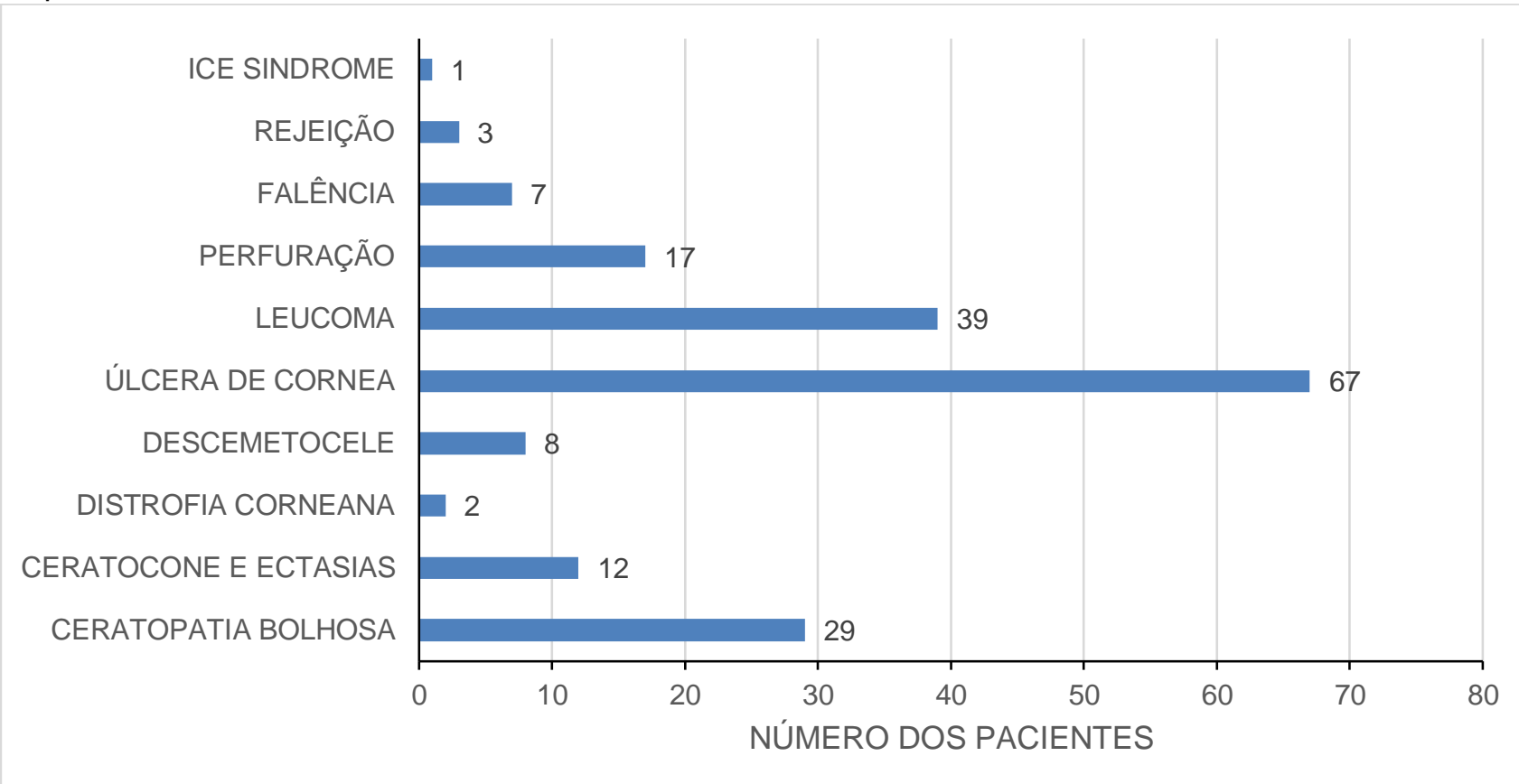

Fonte: Dados da pesquisa.

Já em relação ao tempo de espera nas indicações para o procedimento no critério urgente, observou-se o predomínio dos pacientes submetidos ao transplante num período até 14 dias (43,4\%) (Gráfico 2) e, nas indicações eletivas, houve uma espera média de 835 dias (Gráfico 3).

Gráfico 2 - Tempo de espera para de transplantes de urgência realizados no Hospital Universitário Bettina Ferro de Souza no período de outubro de 2011 a dezembro de 2017.

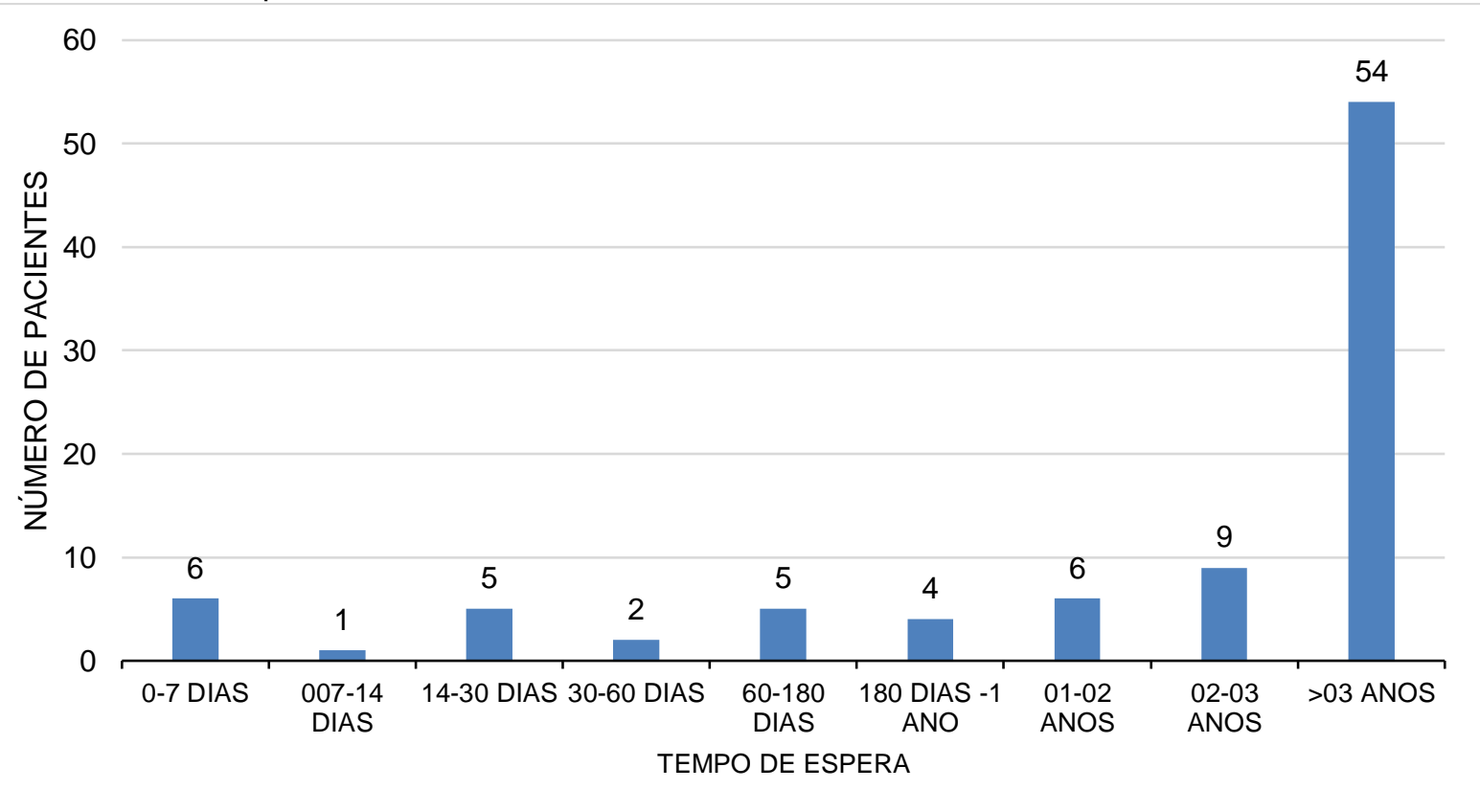

Fonte: Dados da pesquisa, 2019.

REAS/EJCH | Vol. 11 (11) | e419 | DOI: https://doi.org/10.25248/reas.e419.2019 Página 4 de 7 
Gráfico 3 - Tempo de espera para de transplantes eletivos realizados no Hospital Universitário Bettina Ferro de Souza no período de outubro de 2011 a dezembro de 2017.

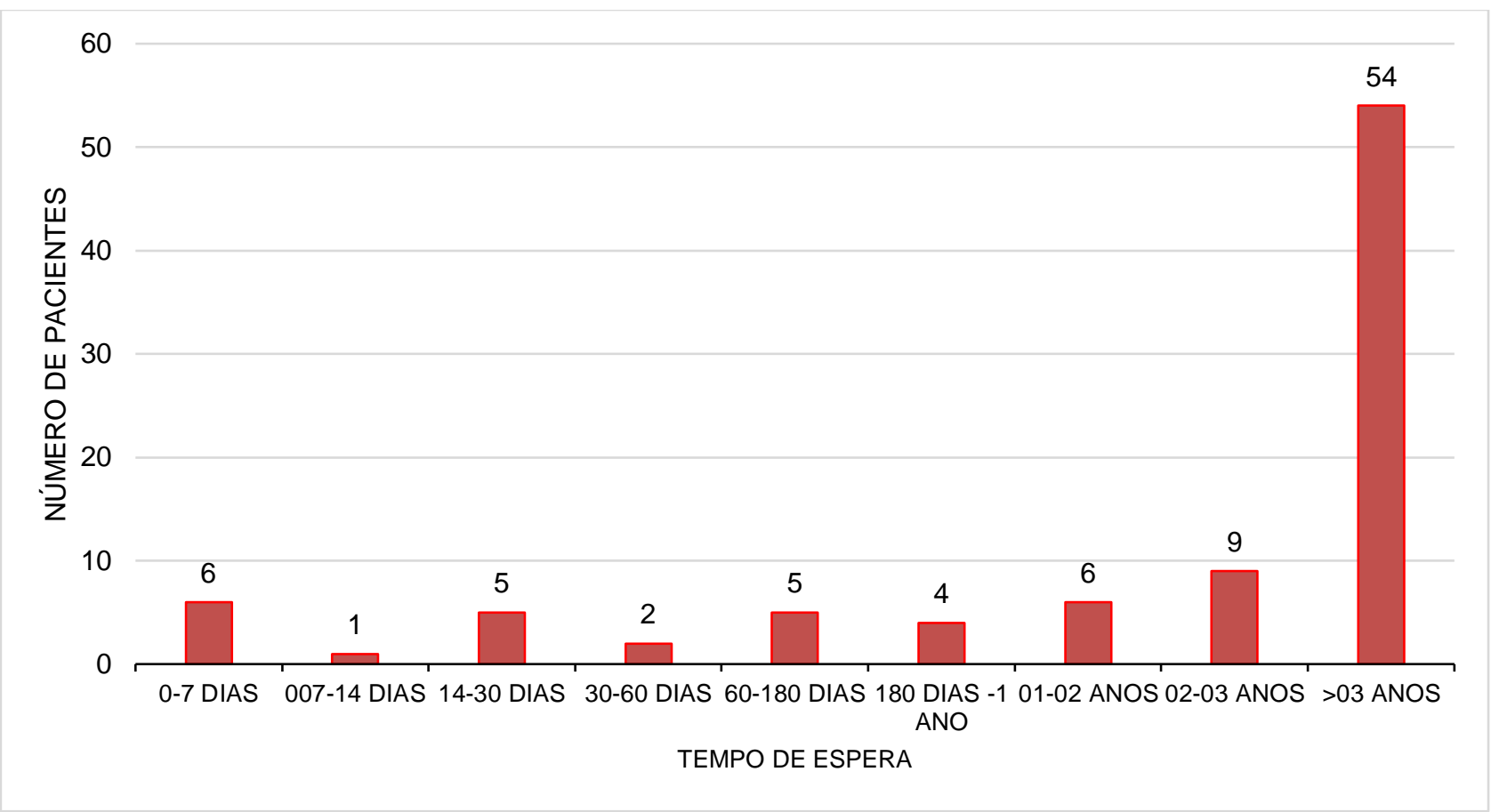

Fonte: Dados da pesquisa, 2019.

Por fim, houve um pequeno predomínio de procedimentos realizados em urgência, em comparação com os eletivos (Gráfico 4).

Gráfico 4 - Tipo de cirurgia realizada no Hospital Universitário Bettina Ferro de Souza no período de outubro de 2011 a dezembro de 2017.

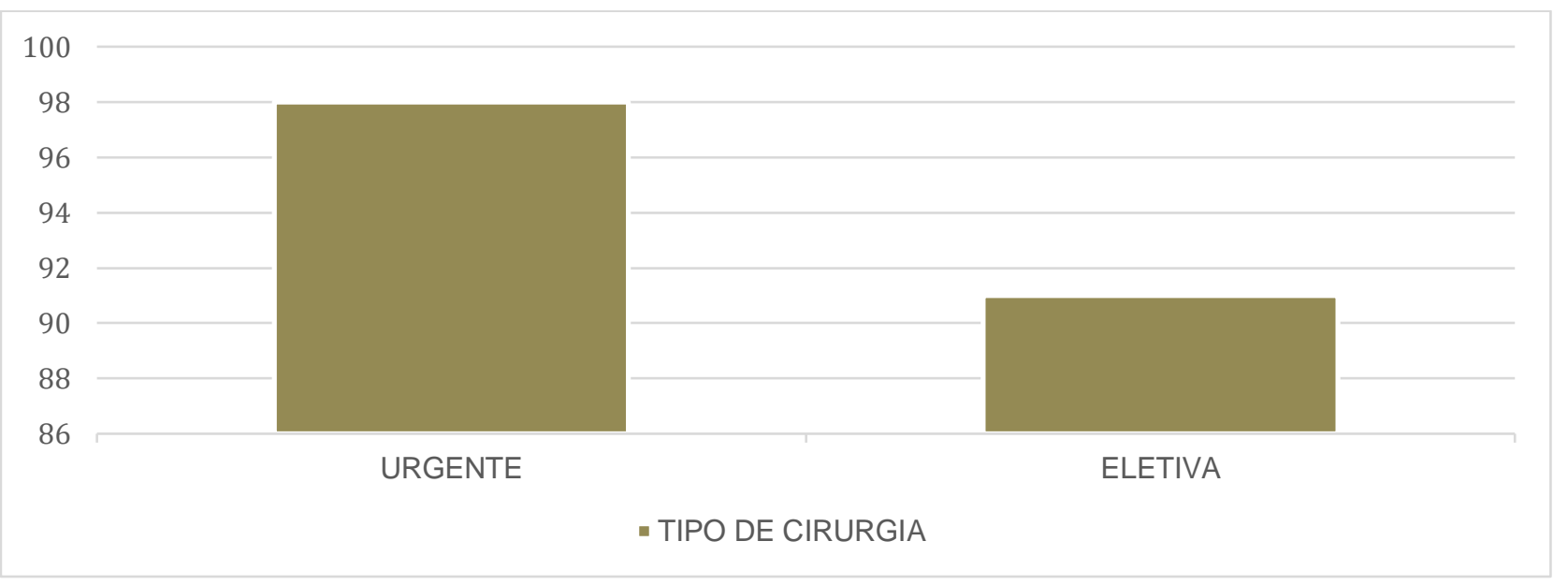

Fonte: Dados da pesquisa, 2019.

\section{DISCUSSÃO}

Foi observado predomínio do sexo masculino dentre todos os pacientes transplantados de córnea no HUBFS, o que contrasta com o estudo de Kara-Junior et al. (2011), no qual o sexo feminino foi o mais frequente. No entanto, há consonância com o estudo de Neves RC et al. (2010), no qual também predominou o gênero masculino. Tal fato pode ser justificado pela alta prevalência de transplantes por indicação de úlcera 
de córnea na amostra do hospital, sendo o gênero masculino o mais acometido dessa afecção. (Neves RC et al., 2010).

Em relação à faixa etária, a maioria foi de pacientes com idade acima de 60 anos, apresentando também um predomínio em paciente das faixas de 31 a 40 e 41 a 50, obtendo-se uma idade média de 46 anos. 0 mesmo padrão foi observado em estudo de hospital de referência para tal procedimento no Estado do Rio Grande do Norte, Nordeste do país, onde se obteve a idade média de 49 anos e com duas frequências modais em indivíduos adultos jovens ( 31 a 50 anos) e indivíduos com idade acima de 60 anos, o que coincide com as frequências de incidência das maiores afecções indicativas de transplante por faixa etária, conforme o presente estudo, a úlcera corneana na faixa mais jovem e o leucoma e a ceratopatia bolhosa em pessoas com idade mais avançada, dessa forma, podendo justificar tal frequência (CRUZ GK, et al., 2016)

Percebe-se um pequeno predomínio de indivíduos do interior do Estado e outras cidades do Norte do Brasil, diferindo-se do estudo de Cruz GK et al. (2017), em que a maioria dos transplantados foi da região urbana do Estado e, principalmente, da capital, sendo a procedência desses indivíduos influenciada pela sua condição ocular indicativa. No estudo de Cruz GK et al. (2016), também realizado no Rio Grande do Norte, houve maior frequência de pessoas da zona rural e do interior nos casos de transplantes de urgência, em que a maior indicação era por úlcera de córnea, tal como a presente pesquisa. Percebe-se então, uma influência das condições indicadores do procedimento na procedência dos pacientes, principalmente por conta da maior chance de trauma corneano grave em zonas rurais onde há pouca utilização de equipamentos de proteção individual pelos trabalhadores rurais.

Úlcera corneana foi a principal indicação para o transplante de córnea, seguida por leucoma e ceratopatia bolhosa. Na literatura mundial, no entanto, observa-se um maior predomínio de indicações por distrofias corneanas, como observado em estudos na Alemanha e no Canadá. Como se pode inferir, há discordância devido, principalmente, à qualidade e à quantidade de serviços de referência oftalmológica nesses países desenvolvidos (SMINKEY L, 2018; BENTLEY TS, et al., 2017).

Já na literatura nacional, observa-se concordância dos dados com a presente pesquisa, sendo que, no estudo de Neves RC et al. (2010), a úlcera de córnea também foi a principal afecção indicadora do transplante, em estudo realizado no Sudeste do país, e na pesquisa de Cruz GK et al. (2017), realizada no Nordeste brasileiro, tal indicação foi a segunda mais frequente. Tais estatísticas nacionais podem ser justificadas pelo maior risco de desenvolvimento de úlcera de córnea em condições de má higiene e maus hábitos e cuidados principalmente no manuseio de lentes de contato, o que se observa mais frequentemente em países em desenvolvimento.

O tempo de espera nas indicações de urgência foi de até 14 dias, o que está de acordo com a literatura nacional, conforme estudos de Cruz GK et al. (2016) e Bonfadini G et al. (2014). Nas indicações eletivas, houve uma espera média de dois anos e dois meses, período maior que na maioria dos Estados brasileiros, em consequência da baixa doação e coleta de córneas na região norte do Brasil, dificultado o acesso e aumentando o período de espera.

Por fim, houve um pequeno predomínio de procedimentos de urgência em comparação com os eletivos, o que é justificado pela principal indicação (úlcera de córnea) dos transplantes realizados no hospital, o que também se observa em estudo de Neves RC et al. (2010) e também pela maior facilidade em aquisição de córneas com padrão para uso tectônico ou terapêutico.

\section{CONCLUSÃO}

Conclui-se, então, que a maioria dos pacientes submetidos ao transplante de córnea no Hospital Bettina Ferro de Souza, no período de outubro de 2011 a dezembro de 2017, foi do gênero masculino, com faixa etária maior que sessenta anos, procedente do interior do Estado, com a principal afecção indicadora sendo a úlcera de córnea, com tempo de espera de até quatorze dias para procedimentos urgentes e média de 2 anos e 2 meses nos eletivos, com maior frequência de transplantes realizados em urgência. Dados estes que contribuem para a formação do perfil de pacientes de tipos de cirurgias realizadas no norte do país. 


\section{REFERÊNCIAS}

1. SMINKEY

L.

Priority

eye

diseases.

2018.

Disponível

em:

$<$ http://www.who.int/blindness/causes/priority/en/index8.htmlWHO>. Acesso em: 25 de maio de 2018.

2. BENTLEY TS, et al. S organ and tissue transplant cost estimates and discussion. Estados Unidos: Milliman research report. 2017.

3. Eye Banking Statistical Report 2015. Washington: Eye Bank Association of America. 2016.

4. CRUZ GK, et al .Epidemiological Profile of Patients Undergoing Urgent Corneal Transplant in a Referral Center in Northeastern Brazil. Transplant Proc. 2016 Sep;48(7):2366-2370.

5. CRUZ GK,et al. Aspectos clínicos e epidemiológicos dos pacientes transplantados com córneas em um serviço de referência. Rev. Latino-Am. Enfermagem vol.25. Ribeirão Preto 2017.

6. NEVES RC, et al. Indicações de transplante de córnea no Hospital São Geraldo da Universidade Federal de Minas Gerais. RevBras Oftalmol. 2010; 69 (2): 84-8.

7. Chen MC, et al. Corneal transplantation at Tenwek Hospital, Kenya, East Africa: Analysis of outcomes and associated patient socioeconomic characteristics. PLOS ONE. Outubro 2017.

8. GAIN P, et al. Global survey of corneal transplantation and eye banking. JAMA Ophthalmol. 2016; 134(2):16773.

9. PINEDA R. Corneal transplantation in the developing world: Lessons learned and meeting the challenge. Cornea. 2015; 34 Suppl 10:S35-40.

10. Transplantes realizados por unidade federativa. Ministério da Saúde. 2018. Disponível em: http://tabnet.datasus.gov.br/cgi/tabcgi.exe?sih/cnv/qiuf.def. Visitado em: 17 de maio de 2018.

11. DEKARIS I. Current Trends in Corneal Transplantation. Rad 517. Medical Sciences, 2013; 35-46.

12. ALMEIDA EFS, et al. Perfil epidemiológico de pacientes na fila de transplante penetrante de córnea no estado do Pará, Brasil. RevBras Oftalmol. 2011; 70 (6): 384-90.

13. KARA-JUNIOR N, et al. Expectativas e conhecimento entre pacientes com indicação de transplante de córnea. Rev Bras Oftalmol. 2011; 70 (4): 230-34

14. BONFADINI G, et al. Doação e fila de transplante de córnea no Estado do Rio de Janeiro. Rev Bras Oftalmol. 2014; 73 (4): 237-42. 